Research Article

\title{
Green Preparation, Spheroidal, and Superior Property of Nano-1,3,5,7-Tetranittro-1,3,5,7-Tetrazocane
}

\author{
Xinlei Jia $(\mathbb{D}$, Jingyu Wang $\mathbb{D}$, Conghua Hou $\mathbb{D}$, and Yingxin Tan $(\mathbb{D}$ \\ School of Chemical and Environmental Engineering, Shanxi Engineering Technology Research Center for Ultrafine Powder, \\ North University of China, Taiyuan, Shanxi 030051, China \\ Correspondence should be addressed to Conghua Hou; houconghua@163.com
}

Received 19 June 2018; Accepted 1 August 2018; Published 18 September 2018

Academic Editor: Chandragiri V. Reddy

Copyright ( $) 2018$ Xinlei Jia et al. This is an open access article distributed under the Creative Commons Attribution License, which permits unrestricted use, distribution, and reproduction in any medium, provided the original work is properly cited.

\begin{abstract}
Herein, a green process for preparing nano-HMX, mechanical demulsification shearing (MDS) technology, was developed. Nano-HMX was successfully fabricated via MDS technology without using any chemical reagents, and the fabrication mechanism was proposed. Based on the "fractal theory," the optimal shearing time for mechanical emulsification was deduced by calculating the fractal dimension of the particle size distribution. The as-prepared nano-HMX was characterized by X-ray diffraction (XRD), scanning electron microscopy (SEM), and differential scanning calorimetry (DSC). And the impact sensitivities of HMX particles were contrastively investigated. The raw HMX had a lower fractal dimension of 1.9273. The ideal shearing time was $7 \mathrm{~h}$. The resultant nano-HMX possessed a particle size distribution ranging from $203.3 \mathrm{~nm}$ to $509.1 \mathrm{~nm}$ as compared to raw HMX. Nano-HMX particles were dense spherical, maintaining $\beta$-HMX crystal form. In addition, they had much lower impact sensitivity. However, the apparent activation energy as well as thermal decomposition temperature of nano-HMX particles was decreased, attributing to the reduced probability for hotspot generation. Especially when the shearing time was $7 \mathrm{~h}$, the activation energy was markedly decreased.
\end{abstract}

\section{Introduction}

HMX has been widely used as the main component of highenergy explosives. It exhibits high energy density, good heat resistance, stable detonation, and high detonation speed. However, high mechanical sensitivity and poor shock wave sensitivity limit its application to a large extent [1]. With the rapid development of modern warfare and the deteriorating battlefield environment, the safety performance for weapons and ammunition becomes ever more critical. Ammunition need not only meet the requirements of long range, high precision, and strong power but also require insensitivity. Therefore, insensitivity has become an important indicator to measure the performance of explosives [2,3]. One of the ways to achieve this goal is the ultrafining technology of explosives. Through refinement, the surface of the explosives becomes smooth, the particle size gets smaller, and the particle morphology tends to be spherical. In this case, the probability of hotspot generation and propagation is reduced, thus achieving desensitization [4].
In recent years, more and more researchers are devoted to the refinement of single explosives, mainly consisting of the nuclear growth method and the ultrafine grinding method to realize the ultrafining treatment. However, these two methods have their own obvious shortcomings. The nuclear growth method is to formulate ultrafine particles of a specified size by controlling conditions for crystal nucleation growth. During the preparation process, high temperatures may be required, which may cause explosive explosion and deflagration. In addition, the explosives refined by this method may undergo crystal transformation, thus affecting the sensitivity. Song et al. [5] used solvent-nonsolvent method to prepare ultrafine HMX. The raw material possessed $\beta$-HMX, but the refined particles contained $\gamma$-HMX, which could reduce the purity of the product. van der Heijden and Bouma [6] spheroidized HMX and RDX through simple mixing and stirring in a special solvent. However, the spheronization effect was not obvious, and the quality of the obtained crystal was poor. An et al. [7] prepared HMX with a particle size distribution of $40 \sim 130 \mathrm{~nm}$ by 
green recrystallization process. The resultant HMX was polyhedron and spherical. However, there was a certain degree of crystal transformation during the recrystallization process, and a large amount of organic solvents were used in the experiment, so this method was not truly "green." Shang and Zhang [8] used different solvents to prepare HMX with different particle sizes by supercritical antisolvent method (GAS) recrystallization method. However, this method had a low yield, and the subsequent processing was troublesome. The ultrafine grinding method is to pulverize large-sized particles by a certain means to reduce the particle size. In the field of energetic materials, there are many reports on the preparation of ultrafine explosive particles via mechanical ball milling. However, there exist certain disadvantages in such process. With the increase of milling time, high temperature and high pressure appear in the barrel of the ball mill. In this way, on the one hand, the safety performance will be reduced; on the other hand, some physicochemical reactions (such as crystal transformation) may occur when the crystal grains are activated [9]. Liu et al [10] prepared nano-RDX by mechanical ball milling, which reduced the impact sensitivity by $30 \%$ and the friction sensitivity by $92.8 \%$, but this method was easy to introduce impurities. Wang et al. [11] prepared HMX by wet milling, solvent-antisolvent, and wet sieve method. For particles obtained by solvent-antisolvent method, the particle size distribution was narrow and the surface was relatively smooth. However, the experiment has poor repeatability and serious environmental pollution.

In this work, based on the shortcomings of the above refinement process, we proposed an improved green approach to refine HMX, i.e., mechanical demulsification shearing (MDS) technology. This technology addressed the unfavorable factors of the existing refinement. First of all, this method effectively solved the problem of the crystal transformation of explosives, because the entire experiment was a physical process and no new substances and impurities were generated. Secondly, the whole process was highly repeatable, and no organic solvents were used throughout the experiment, thus achieving the goal of environmental protection. Finally, to achieve nanometerization, the explosives were refined by relying on mechanical shearing of explosive particles and collisions between particles in a narrow space. Therefore, this technology greatly improved the safety performance during the mechanical ball milling and avoided the introduction of impurities.

\subsection{Experimental Section}

1.1.1. Materials. HMX was provided by Gansu Yinguang Chemical Industry Group Co. Tween-80 and ethanol were from Tianjin Shen Tai Chemical Reagent Co., Ltd. Span-80 was obtained from Tianjin Damao Chemical Reagent Factory. Pure water is provided by pure water supply of Taiyuan Iron and Steel Co., Ltd.

1.1.2. Experimental Process. The experimental procedure for preparing nano-HMX via mechanical demulsification shearing (MDS) technology is as follows:
(1) Preparation of composite emulsifier. Composite emulsifier is obviously superior to single emulsifier in improving the stability of emulsion explosives. Based on this, $2.65 \mathrm{~g}$ Tween-80 and $2.35 \mathrm{~g}$ Span-80 were added to the beaker with $M_{\text {(Tween-80) }}: M_{(\text {Span-80) }}$ of 53:47. Stir for 10 minutes under the action of a magnetic stirrer until the emulsion was completely mixed and then set aside.

(2) Preparation of HMX emulsion. Diluted HMX (10 g) and purified water $(500 \mathrm{ml})$ were added to the beaker, respectively; then, a certain amount of selfmade composite emulsifier was added to the beaker, too. The stirring speed was set as $500 \mathrm{rad} / \mathrm{min}$, and the mixed solution was uniformly stirred for $40 \mathrm{~min}$ at $30^{\circ} \mathrm{C}$. Here, a homogenous HMX emulsion was obtained.

(3) Preparation of nano-HMX by MDS. The beaker containing the HMX emulsion was placed under the emulsifying machine. Then slowly increase the speed to $7500 \mathrm{rad} / \mathrm{min}$ and continue stirring (the reaction process was carried out in a low temperature circulating condensate pump, and the shear temperature was maintained at $40^{\circ} \mathrm{C}$ throughout the experiment). After stirring, the solution was let to stand, followed by filtration and natural drying for 12 hours, affording high-quality nano-HMX particles.

As shown in Figure 1, due to strong fluid shear and severe high-frequency mechanical effects, the HMX emulsion was sucked into the rotor from the bottom of the beaker for strong mixing. Then, it threw from stator gap and hit each other under the action of centrifugal force. Driven by high-speed rotating stator, HMX particles collided with each other in a small space (in the long-hole rotor). And plus the constant shear of the fluid, the nano-HMX was finally obtained. In order to explore the optimal shearing time by MDS, under the same experimental conditions, we cut the as-prepared nano-HMX at different time points of $4 \mathrm{~h}, 5 \mathrm{~h}, 6 \mathrm{~h}, 7 \mathrm{~h}$, and $8 \mathrm{~h}$ and labeled them as nano-HMX1, nano-HMX-2, nano-HMX-3, nano-HMX-4, and nanoHMX-5, respectively.

1.2. Characterization. Field-emission scanning electron microscopy (FESEM) images were taken on a MIRA3 LMH SEM (TESCAN) at $10 \mathrm{k}$. X-ray diffraction (XRD) patterns were obtained using a DX-2700 (Dandong Haoyuan Corporation, Liaoning, China) X-ray diffractometer with $\mathrm{Cu}-\mathrm{K} \alpha$ $(40 \mathrm{kV}, 30 \mathrm{~mA})$ radiation at $\lambda=1.5418 \AA$. All samples were scanned from $5^{\circ}$ to $50^{\circ}$ with steps 0.03 and 6 s counting time. Thermal analysis was performed on a differential scanning calorimeter (DSC-131, France SETARAM Corporation, Shanghai, China) at heating rates of $5,10,15$, and $20^{\circ} \mathrm{C} /$ min. The impact sensitivity was tested with a homebuilt type 12 drop hammer apparatus. The special height (H50) represents the height from which $2.500 \pm 0.002 \mathrm{~kg}$ drop hammer will result in an explosive event in $50 \%$ of the trials. In each determination, 25 drop tests were made to calculate the H50. The particle size tested by QICPIC dynamic particle 


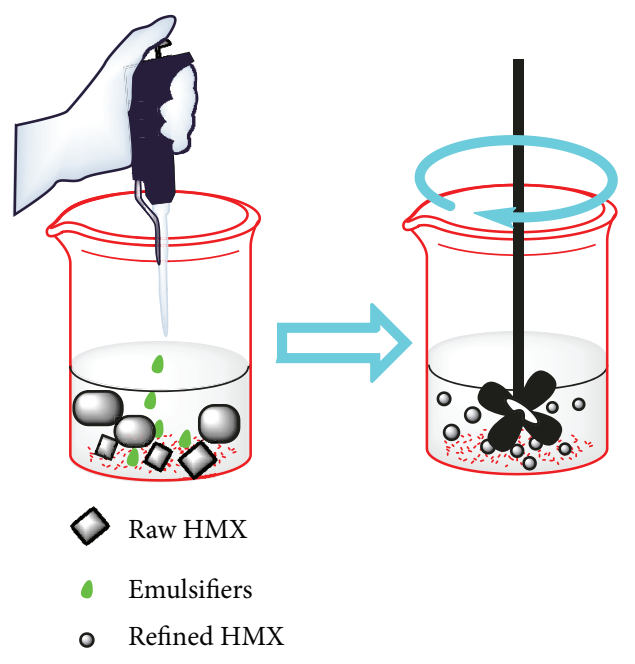

FIGURE 1: Schematic formation of nano-HMX.

analyzer (Sympatec Co., Ltd., Germany), and its working environment is $5 \sim 35^{\circ} \mathrm{C}$, relative humidity is less than $85 \%$, light source type is He-Ne laser, power is $2.0 \mathrm{~mW}$, and wavelength is $0.6328 \mu \mathrm{m}$.

\section{Results and Discussion}

2.1. SEM Images of HMX Samples. Field-emission scanning election microscope (FESEM; HITACHI S4700) was used to display typical SEM images of HMX samples in Figure 2.

As observed in Figure 2, raw HMX is irregular in shape and has a wide and uneven particle size distribution, and there exist cracks. However, nano-HMX prepared via MDS performs better qualities. With the increase of the emulsification shearing time, the particle size gradually becomes smaller, the surface becomes smoother, the particle distribution is more uniform, and the morphology gets close to spherical in shape. Therefore, from the apparent analysis, it contributes to reducing the sensitivity. In particular, nanoHMX as prepared at the emulsification shearing time of $7 \mathrm{~h}$ has the best morphology and particle size distribution. When the shearing time is less than $7 \mathrm{~h}$ (as shown in Figures 2(b)-2(d)), the distribution of HMX particles is noticeably uneven, and there are particles with large morphology. While the shearing time is greater than $7 \mathrm{~h}$ (Figure 2(f)), the particles are severely agglomerated. This can be explained by the granularity fractal theory [12] of nano-HMX particles. The analysis of the particle size distribution of raw HMX and nano-HMX as prepared by MDS shows that the refined nano-HMX particle size distribution is unimodal (Figure 3), which is in good accordance with the granularity fractality of powder particles [13]. In other words, the particle size distribution of HMX has the fractal feature, which satisfies formula (1):

$$
Y \omega(d) \infty d^{3-D},
$$

where $d$ is the particle diameter, $Y \omega(d)$ is the ratio of the total mass of particles less than $d$ to the total mass of all particles in the particle system, and $D$ is the fractal dimension.
According to formula (1), if there is a linear correlation between $\ln Y \omega(d)$ and $\ln d$, it means that the particle size distribution of the powder has fractal characteristics. If the straight line slope $k$ is obtained, the fractal dimension of the particle size distribution can be calculated as $D=3-k$. And the fractal analysis of the particle size distribution of nano-HMX particles is further performed, listed in Table 1.

As Table 1 represents, as the shearing time increases, the particle size of nano-HMX gradually decreases. The correlation coefficient of the straight line of the double logarithmic coordinate system is as high as 0.9898-0.9976, which indicates that the particle size distribution of the raw HMX and the refined HMX has fractal phenomenon. And the particle size distribution of raw HMX has a lower fractal dimension of only 1.9273 , which is easily broken. When the shearing time is $4 \sim 7 \mathrm{~h}$, the particle size distribution dimension of nano-HMX gradually increases. While when the shearing time is $6 \sim 7 \mathrm{~h}$, the fractal dimension decreases slightly, attributing to severe agglomeration between ultrafine particles. And the density of agglomerated particles is slightly lower than that of other particles, thus affecting the proportional relationship in formula (1). From the above discussion, we can see that when the shearing time is $7 \mathrm{~h}$, the resultant nano-HMX has a narrow particle size distribution and smooth surface, and the particle agglomeration is not obvious. Therefore, the shearing time of $7 \mathrm{~h}$ is the best time to fabricate nano-HMX via MDS method.

2.2. Crystal Structure of HMX Samples. XRD analysis was carried out to investigate whether the phase transformation of HMX occurred. X-ray diffraction of HMX samples is displayed in Figure 4:

As illustrated in Figure 4, using the JADE software, nanoHMX (JCPDS no. 00-044-1620) has the same crystal structure as raw $\mathrm{HMX}$ at $2 \theta$ of $12.32^{\circ}, 14.17^{\circ}, 19.25^{\circ}, 22.11^{\circ}$, and $32.27^{\circ}$. It reveals that the crystal structure of the refined HMX is consistent with that of the raw HMX, both maintaining $\beta$-form. The peak of the nano-HMX is much weaker and wider than that of the original HMX, which can be explained by the Scherrer formula (formula 2). The particle size of the particles is inversely proportional to the peak width of the diffraction peak. And it is precisely because the size of ultrafine HMX becomes smaller, resulting in widening of diffraction peaks $[14,15]$. Also, this phenomenon indicates that the HMX crystals are nanosized.

$$
D_{\mathrm{hkl}}=k \frac{\lambda}{\beta} \cos \theta,
$$

where $D_{\text {hkl }}$ is the grain diameter perpendicular to the crystal plane (hkl), $k$ is the Scherrer constant (usually 0.89 ), $\lambda$ is the incident X-ray wavelength (wavelength is $1.5418 \AA$ ), $\theta$ is the Bragg diffraction angle $\left({ }^{\circ}\right)$, and $\beta$ is the peak width of diffraction peak at half height (rad).

2.3. DSC Analysis of Different HMX Particles. Thermal stability is widely considered as a key performance for 


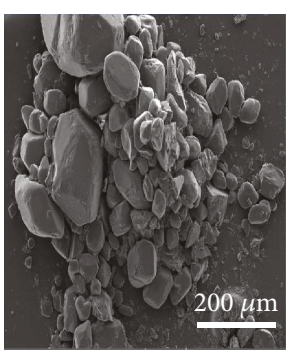

(a)

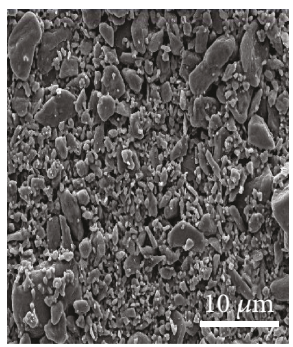

(d)

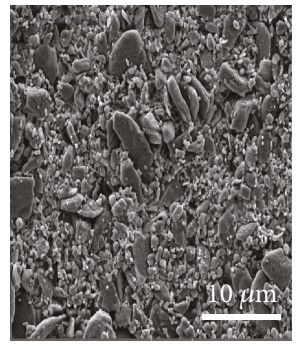

(b)

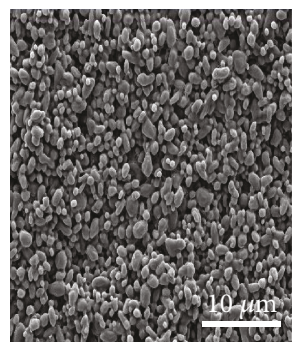

(e)

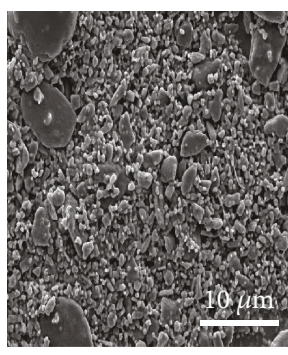

(c)

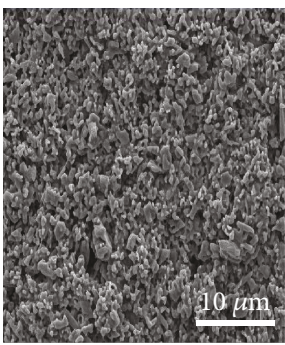

(f)

FIGURE 2: Displays typical SEM images of the HMX samples before and after refined.

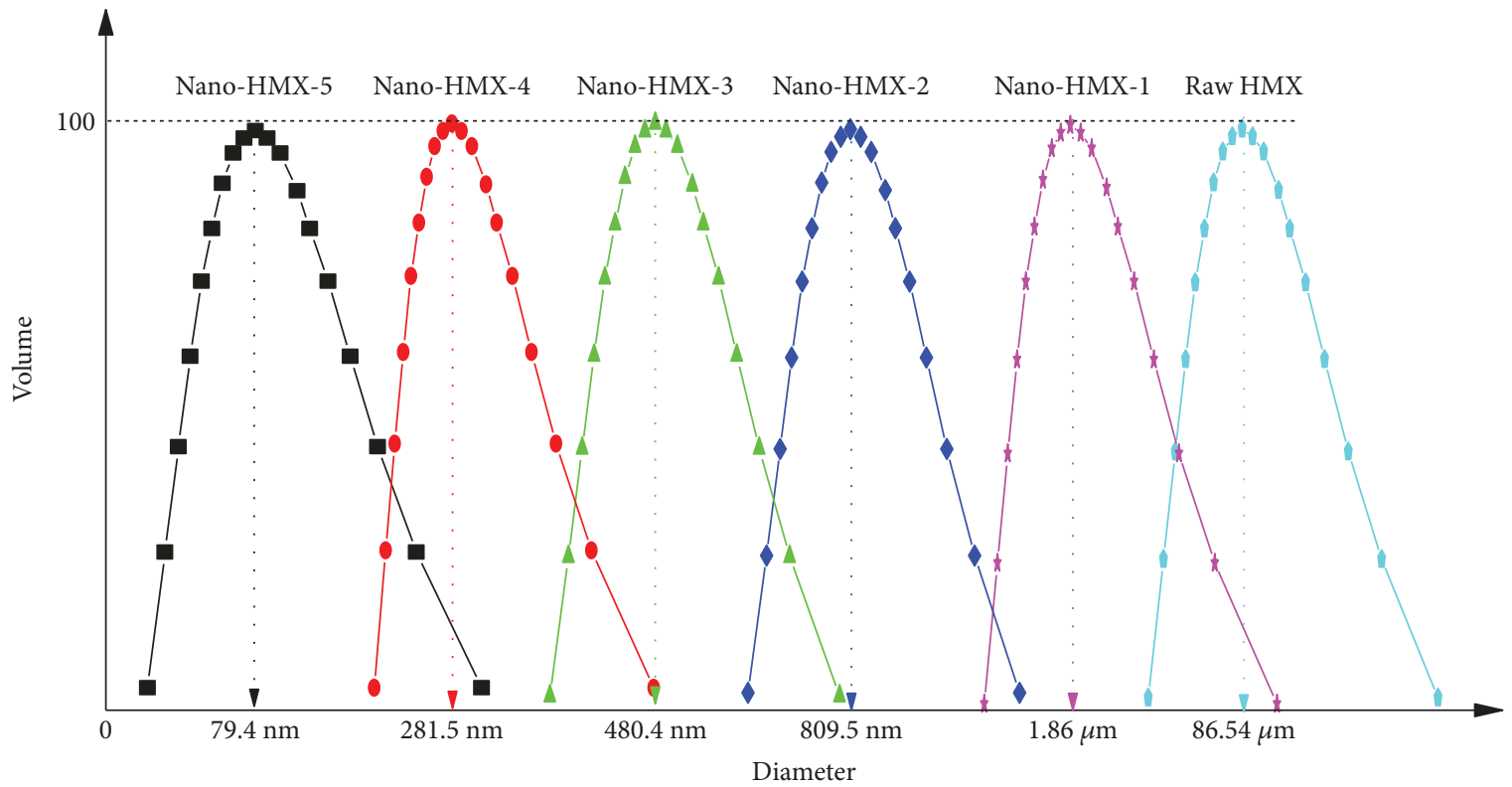

FIgURe 3: Particle size and size distribution of HMX particles.

TABLE 1: Fractal geometry analysis data of HMX particles.

\begin{tabular}{lcccc}
\hline Samples & Shearing time/h & Mean diameter & The dispersion dimension & Correlation coefficients \\
\hline Raw HMX & 0 & $86.54 \mu \mathrm{m}$ & 1.9273 & 0.9938 \\
Nano-HMX-1 & 4 & $1.86 \mu \mathrm{m}$ & 2.7499 & 0.9989 \\
Nano-HMX-2 & 5 & $809.5 \mathrm{~nm}$ & 2.7534 & 0.9898 \\
Nano-HMX-3 & 6 & $480.4 \mathrm{~nm}$ & 2.8497 & 0.9971 \\
Nano-HMX-4 & 7 & $281.5 \mathrm{~nm}$ & 2.9600 & 0.9914 \\
Nano-HMX-5 & 8 & $79.4 \mathrm{~nm}$ & 2.3501 & 0.9976 \\
\hline
\end{tabular}




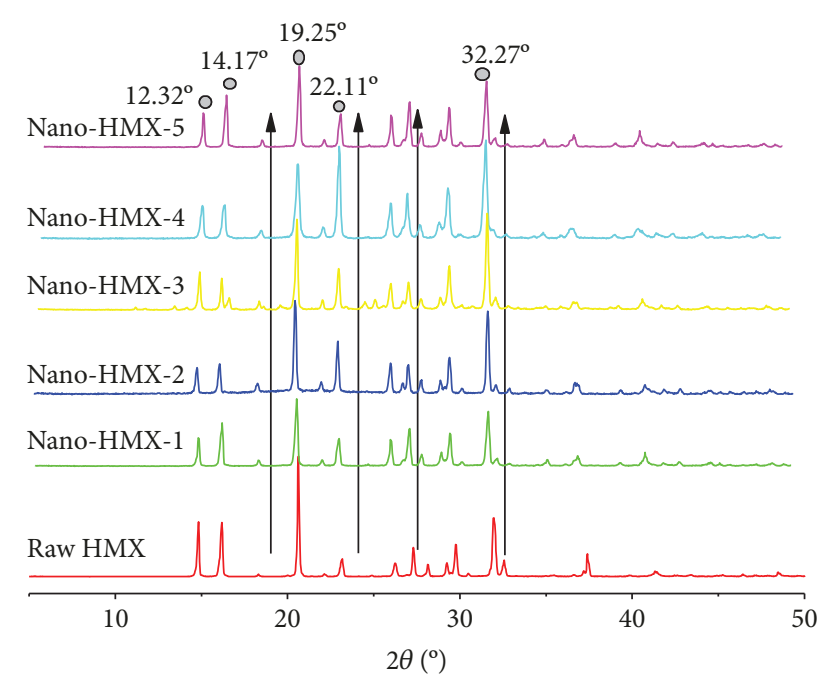

FIgURE 4: XRD spectra of HMX particles.

energetic materials [16]. In our paper, thermal properties of raw HMX and nano-HMX were analyzed by DSC, and their activation energy was calculated. And the changes of thermal properties before and after refinement were compared.

Figure 5 shows that for different heating rates, the decomposition peak temperatures of raw HMX and nano-HMX increase with the enhancement of the heating rate. By Kissinger equation [17-20] (3), from the four exothermic peaks at heating rates of $5 \mathrm{~K} / \mathrm{min}, 10 \mathrm{~K} / \mathrm{min}, 15 \mathrm{~K} / \mathrm{min}$, and $20 \mathrm{~K} /$ $\mathrm{min}$, their apparent activation energy and preexponential factor can be calculated, respectively.

$$
\ln \left(\frac{\beta_{i}}{T_{p i}{ }^{2}}\right)=\ln \left(\frac{A R}{E_{a}}\right)-\frac{E_{a}}{R T_{p i}},
$$

where $\beta_{i}$ is the heating rate $(\mathrm{K} / \mathrm{min}) ; T_{p i}$ is the decomposition peak temperature of the explosive at different heating rates; $A$ is the preexponential factor $\left(\mathrm{min}^{-1}\right) ; R$ is the gas constant, $8.314 \mathrm{~J} /(\mathrm{mol} \cdot \mathrm{K})$; and $E_{a}$ is the apparent activation energy.

As shown in Figure 6, taking $1 / T_{p}$ as the abscissa and ln $\left(\beta / T_{p}{ }^{2}\right)$ as the ordinate, we performed linear fitting on the measurement results and found that the fitting coefficient $R$ is as high as 0.99537 0.99911. Using $E_{a}$ and 4, when the heating rate of HMX approaches 0 , the peak temperature $T_{p 0}$ can be obtained. Then, through the calculation formula (5), the critical temperature of thermal explosion $T_{b}$ can be calculated. And the results are listed in Table 2.

$$
\begin{aligned}
T_{p i} & =T_{p 0}+b \beta_{i}+c \beta_{i}^{2}, \\
T_{b} & =\frac{E-\sqrt{E^{2}-4 R E T_{p 0}}}{2 R} .
\end{aligned}
$$

It can be seen from Table 2 and Figure 7 that the $E_{a}$ and thermal decomposition peak temperatures of the nano-
HMX particles are lower than those of the raw HMX, indicating that nano-HMX is more susceptible to decomposition than raw HMX under thermal stimuli. When the shearing time reaches $8 \mathrm{~h}, E_{a}$ decreases from 485.28 to $427.84 \mathrm{~kJ} /$ mol, and the thermal stability of HMX decreases significantly. Such change can be attributed to the fact that for the same quality HMX, the smaller the particle size is, the larger the surface area will be. At a certain heating rate, the external energy absorbed by the particles increases for a period of time, and the heated area and the reactivity increase. In addition, owing to the massive agglomeration of particles, its specific surface area and the number of atoms located on the surface increase $[21,22]$. Therefore, $E_{a}$ and the thermal decomposition temperature have decreased as compared to the raw HMX.

2.4. The Impact Sensitivity Analysis. Impact sensitivity is also a key parameter to evaluate the safety performance of energetic materials. The impact sensitivities of the HMX samples were separately tested according to GJB 722 A-1997 method 610.302 tool. The impact sensitivity test results are shown in Figure 8 .

As illustrated in Figure 8, compared with the raw material, the $\mathrm{H} 50$ of nano-HMX particles gradually increases with the increase of the shearing time. This can be explained by the hotspot theory [23-25]. On the one hand, as the shearing time increases, the appearance of the HMX particles gradually becomes smoother, and the internal defects gradually decrease, so that the heat transfer rate between the particles increases. When subjected to external forces, it is difficult to form hotspots. On the other hand, for HMX of the same quality, the smaller the particle size is, the more effective the spheroidization will be. As a result, the specific surface area and the gap between the particles increase, resulting the decreased pressure from which the HMX will suffer for the same height falling ball. Therefore, the characteristic height $\mathrm{H} 50$ of nano-HMX increases visibly, thus improving the safety performance.

\section{Conclusion}

The superiority of nano-HMX fabricated by mechanical demulsification shearing (MDS) technology was demonstrated, namely, green preparation, spherical shape, and high-quality properties. More importantly, this method solves the defects of the conventional technology when refining HMX, such as crystal transformation, impurity generation, troublesome follow-up processing, complicated process, and serious pollution. In the paper, nano-HMX was prepared in this way. Based on the experimental analysis, the mechanical emulsification shearing (MDS) technology was proposed through the "fractal theory." The best shearing time for preparing nano-HMX was $7 \mathrm{~h}$. Under this condition, the as-prepared particles tended to possess a spherical morphology and uniform particle size distribution. And no crystal transformation occurred. Compared with the raw HMX, the resultant HMX particles will have better safety performance. However, the apparent activation energy and the thermal decomposition temperature of nano-HMX particles can be decreased, 


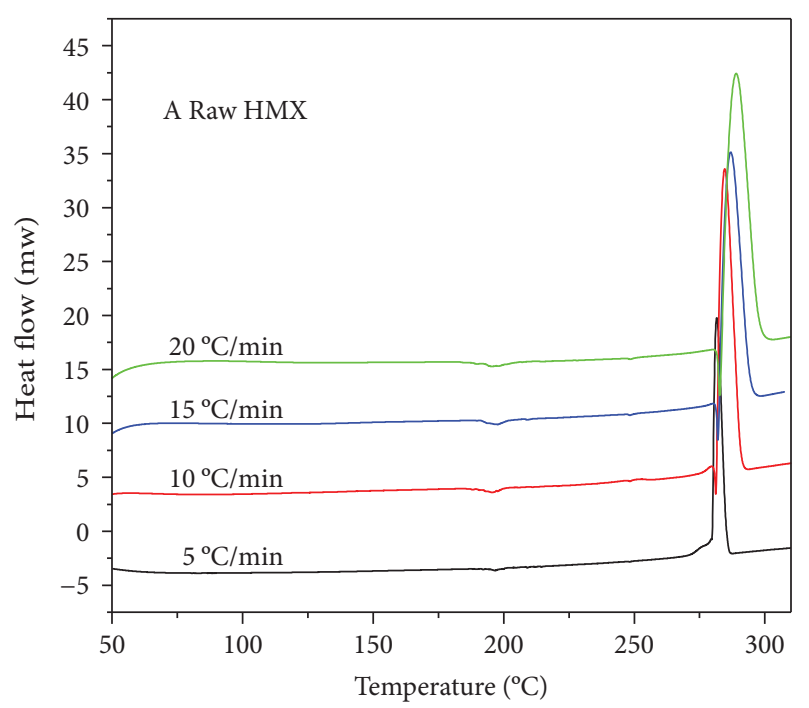

(a)

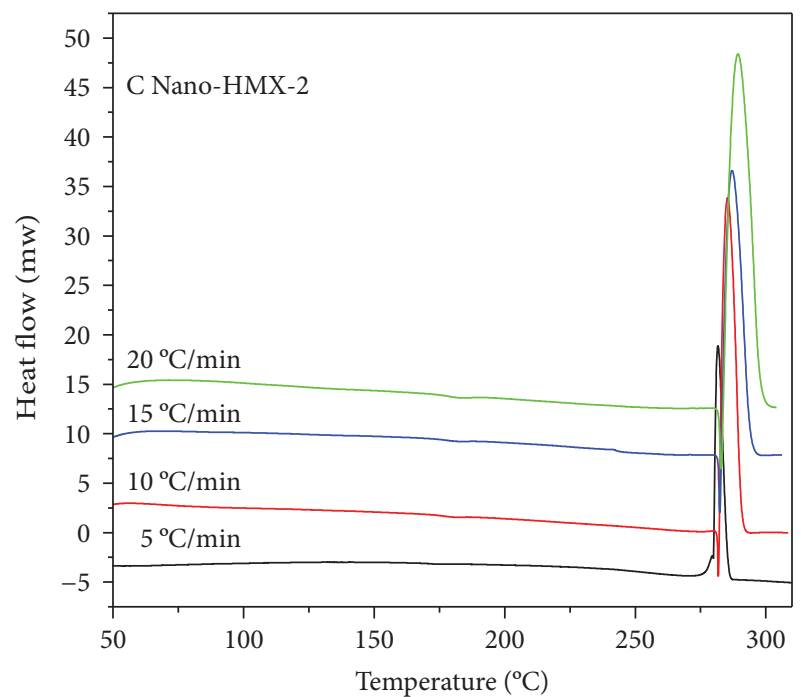

(c)

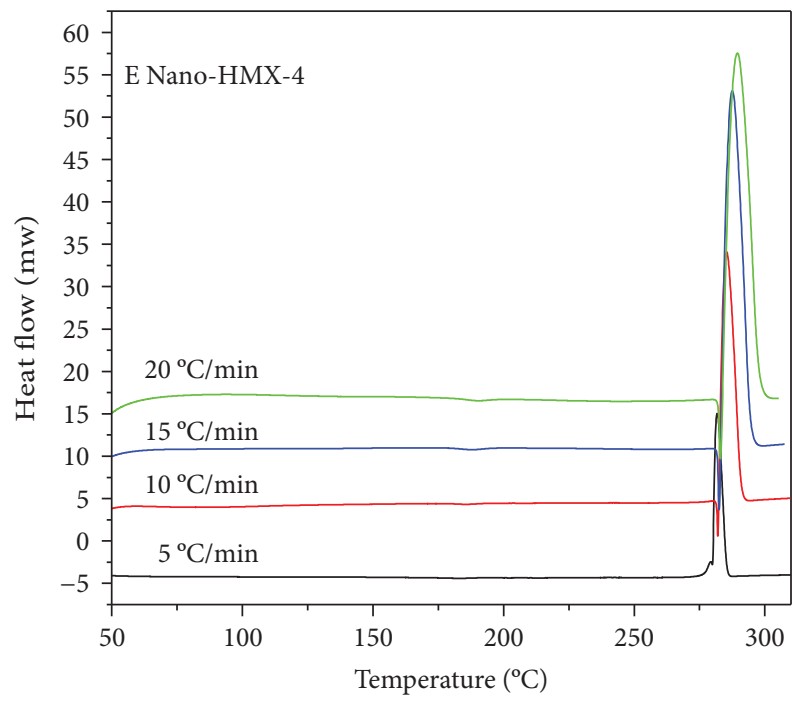

(e)

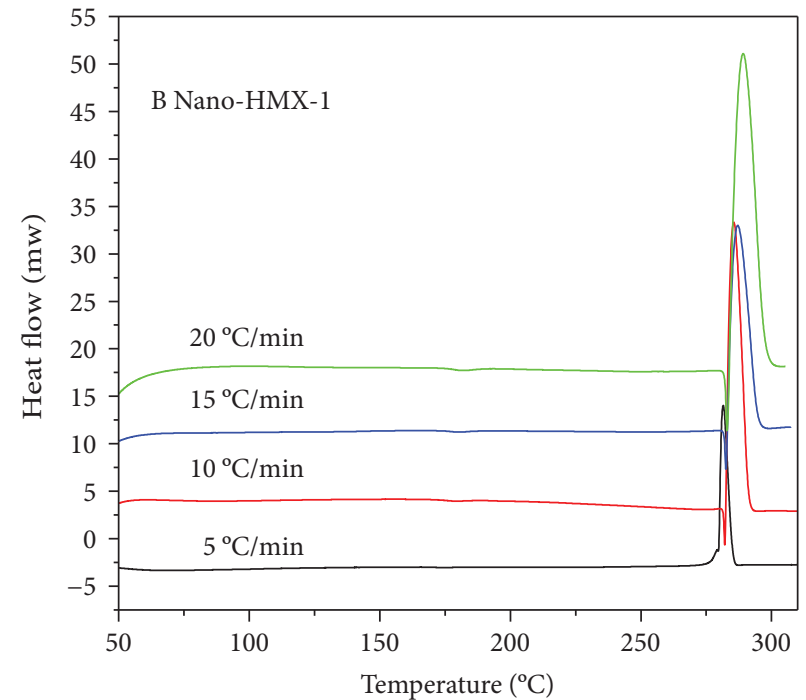

(b)

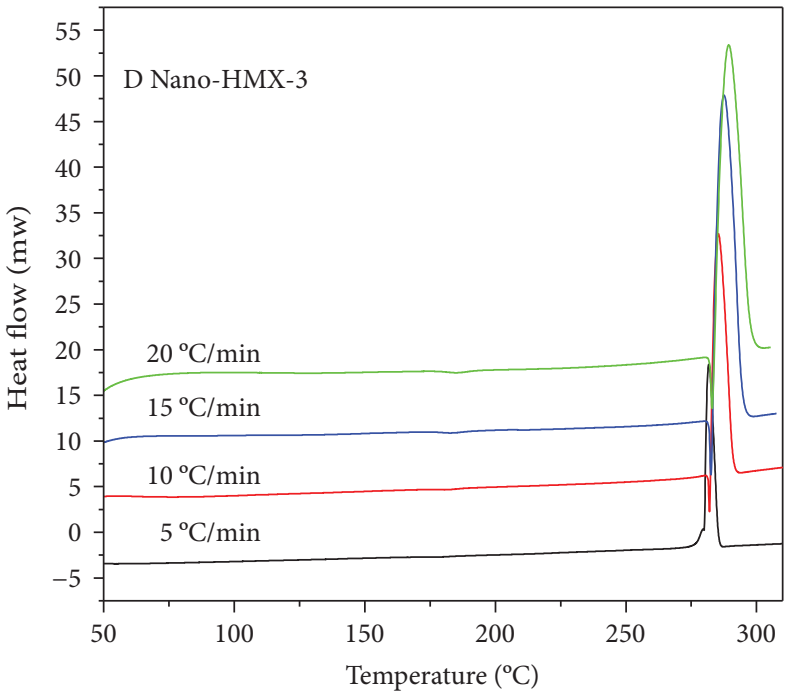

(d)

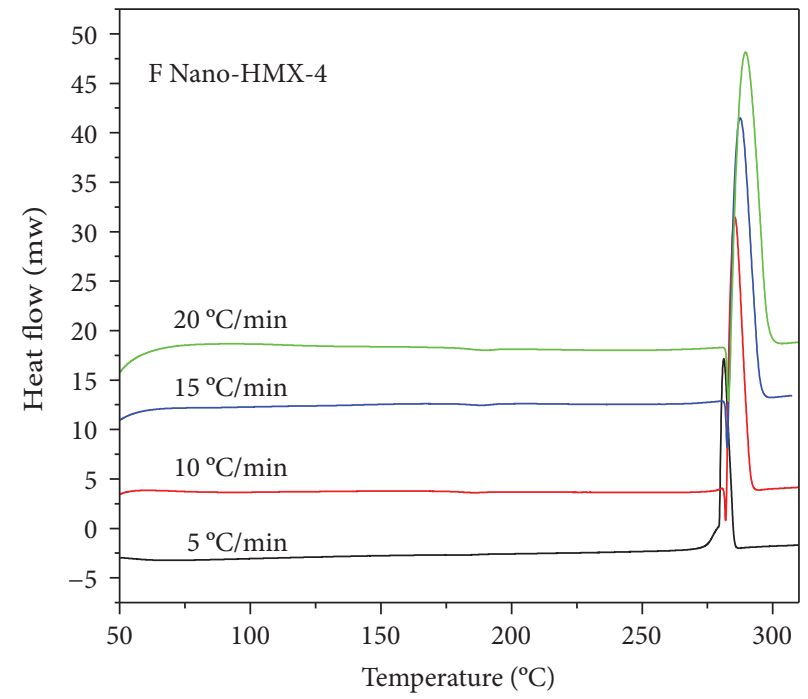

(f)

FIgURE 5: DSC curves of HMX particles. 


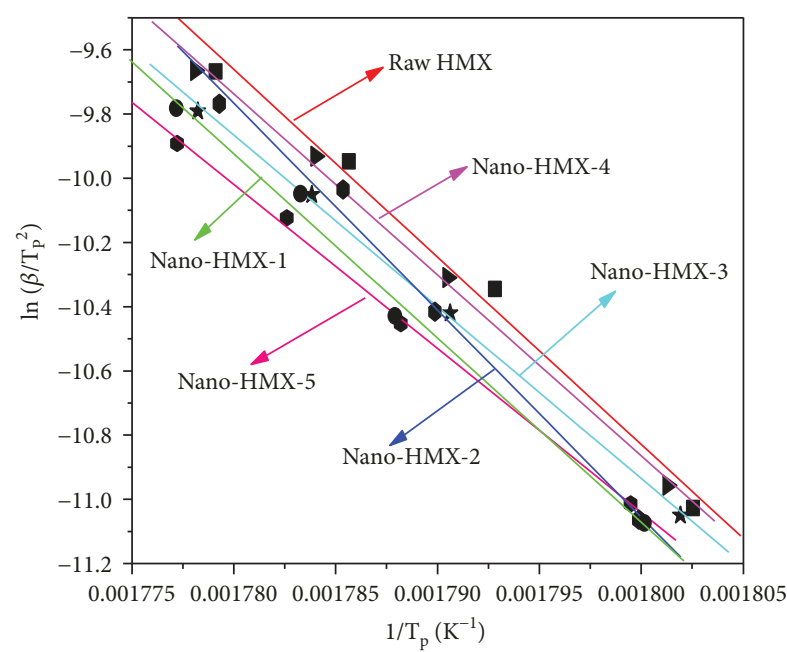

FIgURE 6: Kissinger's plots of $\ln \left(\beta / T_{p}{ }^{2}\right)$ vs. reciprocal peak temperature $1 / T_{p}$ for raw HMX and nano-HMX. Symbol $R$ is used to identify the linear correlation coefficient of $\ln \left(\beta / T_{p}{ }^{2}\right)$ to $1 / T_{p}$.

TAble 2: Thermal decomposition kinetic parameters of different HMX samples.

\begin{tabular}{lcccc}
\hline Samples & $E_{a} / \mathrm{kJ} \cdot \mathrm{mol}^{-1}$ & $\log \left(\mathrm{A} \cdot \mathrm{S}^{-1}\right)$ & $T_{b} /{ }^{\circ} \mathrm{C}$ & $T_{p 0} /{ }^{\circ} \mathrm{C}$ \\
\hline Raw HMX & 485.28 & 45.69 & 278.68 & 277.35 \\
Nano-HMX-1 & 470.00 & 44.23 & 276.60 & 275.25 \\
Nano-HMX-2 & 468.50 & 44.08 & 277.35 & 275.98 \\
Nano-HMX-3 & 468.01 & 44.03 & 277.82 & 276.45 \\
Nano-HMX-4 & 460.79 & 43.34 & 277.92 & 276.53 \\
Nano-HMX-5 & 427.84 & 10.24 & 276.41 & 274.93 \\
\hline
\end{tabular}

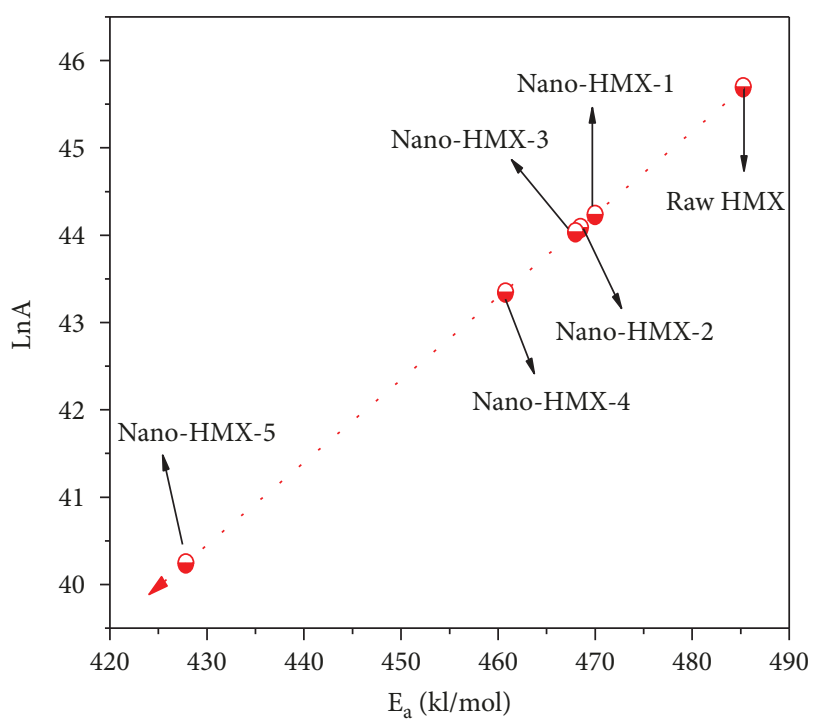

Figure 7: Kinetic compensation effect for thermal decomposition of HMX samples.

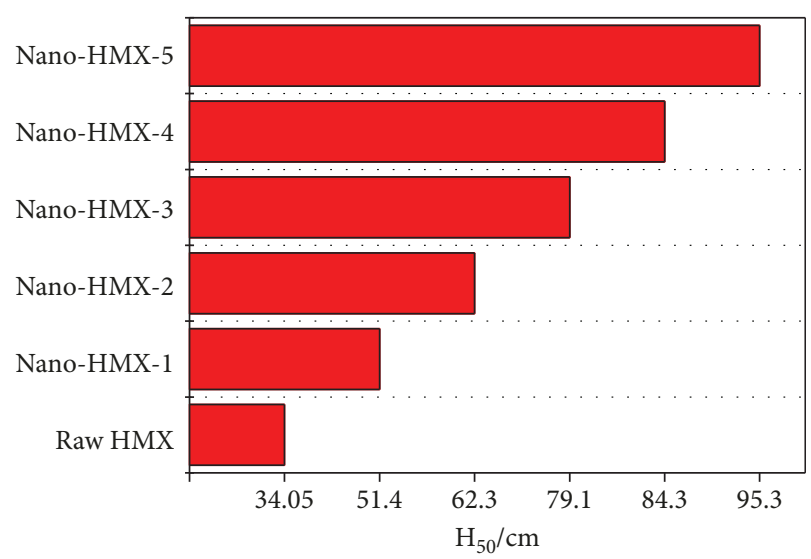

FIGURE 8: Impact sensitivities of HMX samples.

attributing to the reduced hotspot generation probability of the refined particles.

\section{Data Availability}

The data used to support the findings of this study are available from the corresponding author upon request.

\section{Conflicts of Interest}

The authors declare that they have no conflicts of interest.

\section{References}

[1] X. Mao, L. Jiang, C. Zhu, and X. Wang, "Effects of aluminum powder on ignition performance of RDX, HMX, and CL-20 explosives," Advances in Materials Science and Engineering, vol. 2018, Article ID 5913216, 8 pages, 2018.

[2] D. M. Badgujar, M. B. Talawar, S. N. Asthana, and P. P. Mahulikar, "Advances in science and technology of modern energetic materials: an overview," Journal of Hazardous Materials, vol. 151, no. 2-3, pp. 289-305, 2008.

[3] S. M. Walley, J. E. Field, and M. W. Greenaway, "Crystal sensitivities of energetic materials," Materials Science and Technology, vol. 22, no. 4, pp. 402-413, 2006.

[4] H. Liu, G. Deng, Y. Yang, and F. N. Li, "Study on the superfining of explosive by LS superfine pulverizers," Explosive Materials, vol. 33, no. 5, pp. 32-35, 2004.

[5] X. Song, Y. Wang, C. An, X. Guo, and F. Li, "Dependence of particle morphology and size on the mechanical sensitivity and thermal stability of octahydro-1,3,5,7-tetranitro-1,3,5,7tetrazocine," Journal of Hazardous Materials, vol. 159, no. 2-3, pp. 222-229, 2008.

[6] A. E. D. M. van der Heijden and R. H. B. Bouma, "Crystallization and characterization of RDX, HMX, and CL-20," Crystal Growth \& Design, vol. 4, no. 5, pp. 999-1007, 2004.

[7] C. An, H. Li, W. Guo, X. Geng, and J. Wang, "Nano cyclotetramethylene tetranitramine particles prepared by a green recrystallization process," Propellants Explosives Pyrotechnics, vol. 39, no. 5, pp. 701-706, 2015.

[8] F. F. Shang and J. L. Zhang, "Effect of solvent on particle morphology and crystal phase in recrystallization of HMX by different supercritical carbon dioxide as antisolvent," Initiators \& Pyrotechnics, vol. 1, pp. 16-20, 2014. 
[9] M. L. Panchula and J. Y. Ying, "Mechanical synthesis of nanocrystalline $\alpha-\mathrm{Al}_{2} \mathrm{O}_{3}$ seeds for enhanced transformation kinetics," Nanostructured Materials, vol. 9, no. 1-8, pp. 161164, 1997.

[10] J. Liu, L. I. Qing, and J. Zeng, "Mechanical pulverization for the production of sensitivity reduced nano-RDX," Explosive Materials, vol. 42, no. 4, pp. 1-4, 2013.

[11] Y. Wang, X. Song, D. Song, W. Jiang, H. Liu, and F. Li, "Dependence of the mechanical sensitivity on the fractal characteristics of octahydro-1,3,5,7-tetranitro-1,3,5,7-tetrazocine particles," Propellants, Explosives, Pyrotechnics, vol. 36, no. 6, pp. 505-512, 2011.

[12] Y. Liu, Z. Wang, and T. Chai, "Influence of HMX particle size and gradation on the shock sensitivity and output of a PBX explosive," Acta Armamentarii, vol. 4, pp. 357-360, 2000.

[13] X. L. Song, X. D. Guo, J. L. Zhang, C.-W. An, and F.-S. Li, "Dependence of size and size distribution on safety performance of nitroamine explosives and the multi-component explosives," Initiators \& Pyrotechnics, vol. 16, no. 4, pp. 1721, 2007.

[14] C. An, P. Ding, B. Ye, X. Geng, and J. Wang, "Carboncoated copper nanoparticles prepared by detonation method and their thermocatalysis on ammonium perchlorate," AIP Advances, vol. 7, no. 3, article 035324, 2017.

[15] J. Liu, J. Wei, F. Li et al., "Effect of drying conditions on the particle size, dispersion state, and mechanical sensitivities of nano HMX," Propellants, Explosives, Pyrotechnics, vol. 39, no. 1, pp. 30-39, 2014.

[16] Q. L. Yan and S. Zeman, "Theoretical evaluation of sensitivity and thermal stability for high explosives based on quantum chemistry methods: a brief review," International Journal of Quantum Chemistry, vol. 113, no. 8, pp. 1049-1061, 2013.

[17] H. E. Kissinger, "Reaction kinetics in differential thermal analysis," Analytical Chemistry, vol. 29, no. 11, pp. 1702-1706, 1957.

[18] W. Sha, "Determination of activation energy of phase transformation and recrystallization using a modified Kissinger method," Metallurgical and Materials Transactions A, vol. 32, no. 11, pp. 2903-2904, 2001.

[19] M. F. Foltz, C. L. Coon, F. Garcia, and A. L. Nichols, "The thermal stability of the polymorphs of hexanitrohexaazaisowurtzitane, part I," Propellants, Explosives, Pyrotechnics, vol. 19, no. 1, pp. 19-25, 1994.

[20] B. Ye, C. An, Y. Zhang, C. Song, X. Geng, and J. Wang, “Onestep ball milling preparation of nanoscale CL-20/graphene oxide for significantly reduced particle size and sensitivity," Nanoscale Research Letters, vol. 13, no. 1, p. 42, 2018.

[21] C. Hou, X. Jia, J. Wang, Y. Tan, Y. Zhang, and C. Li, "Efficient preparation and performance characterization of the HMX/ $\mathrm{F}_{2602}$ microspheres by one-step granulation process," Journal of Nanomaterials, vol. 2017, Article ID 3607383, 7 pages, 2017.

[22] C. H. Hou, X. L. Jia, J. Y. Wang, and X. M. Jiang, "Preparation of refinement HMX by non-solvent and its performance characterization," Chinese Journal of Explosives \& Propellants, vol. 39, no. 4, pp. 27-31, 2016.

[23] X. L. Jia, C. H. Hou, Y. X. Tan, J. Y. Wang, and B. Y. Ye, "Fabrication and characterization of PMMA/HMX-based microcapsules via in situ polymerization," Central European Journal of Energetic Materials, vol. 14, no. 3, pp. 559-572, 2017.
[24] Z. Li, Y. Wang, Y. Zhang, L. Liu, and S. Zhang, "CL-20 hosted in graphene foam as a high energy material with low sensitivity," RSC Advances, vol. 5, no. 120, pp. 98925-98928, 2015.

[25] Y. Wang, X. Song, D. Song, L. Liang, C. An, and J. Wang, "Synthesis, thermolysis, and sensitivities of HMX/NC energetic nanocomposites," Journal of Hazardous Materials, vol. 312, pp. $73-83,2016$. 


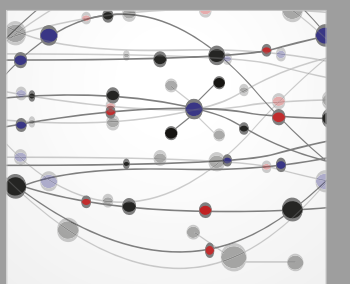

The Scientific World Journal
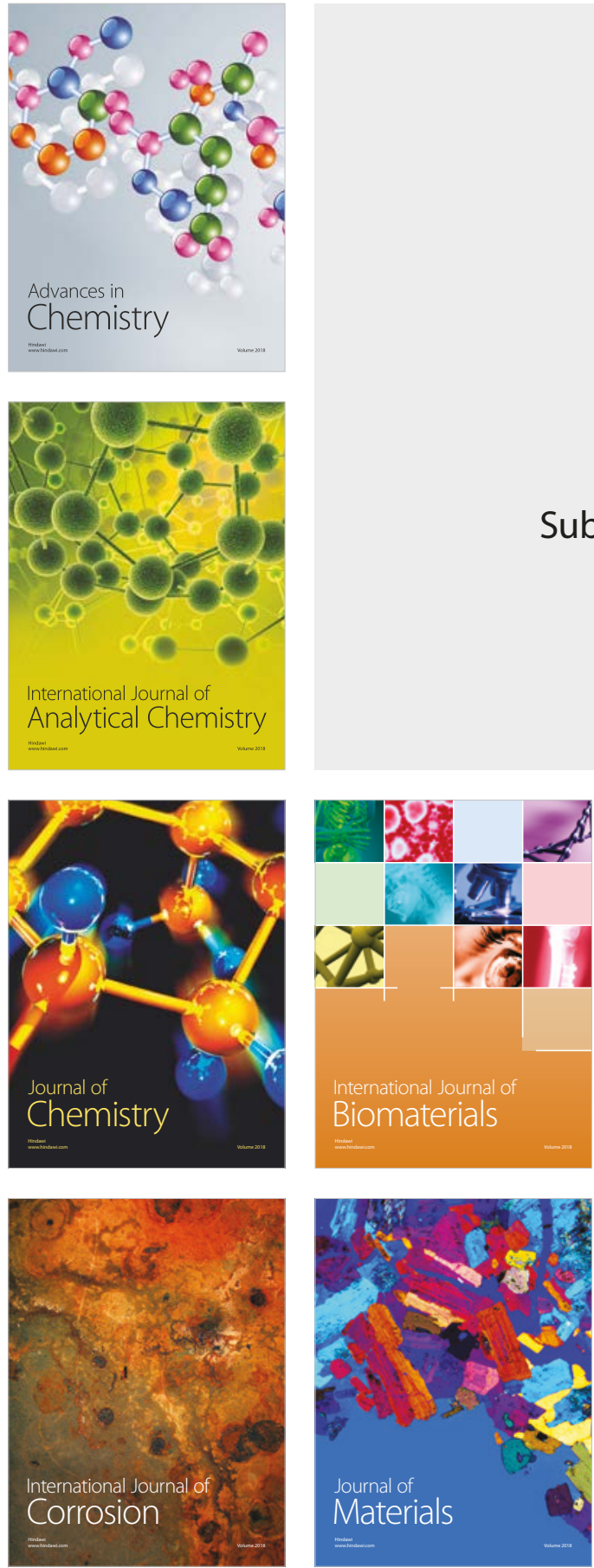

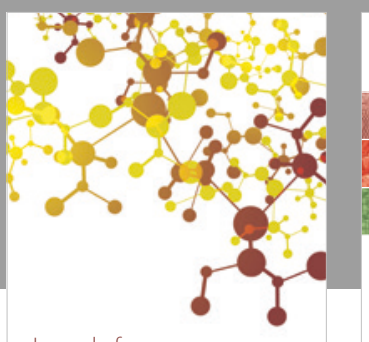

Journal of

Applied Chemistry
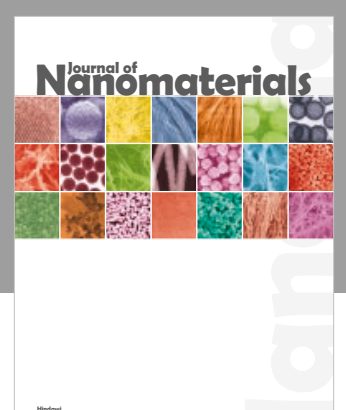

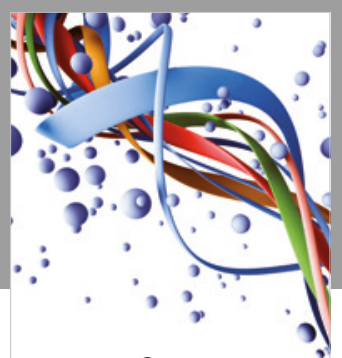

Scientifica

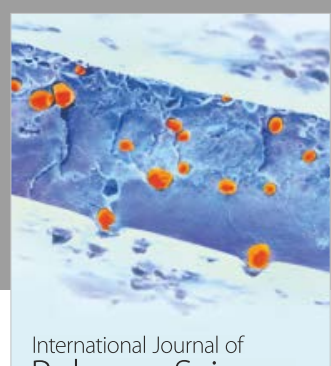

Polymer Science

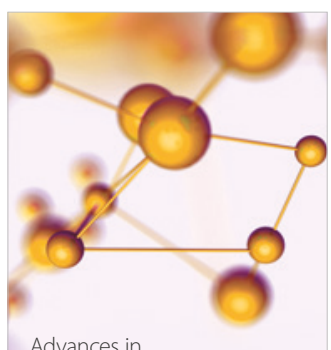

Physical Chemistry
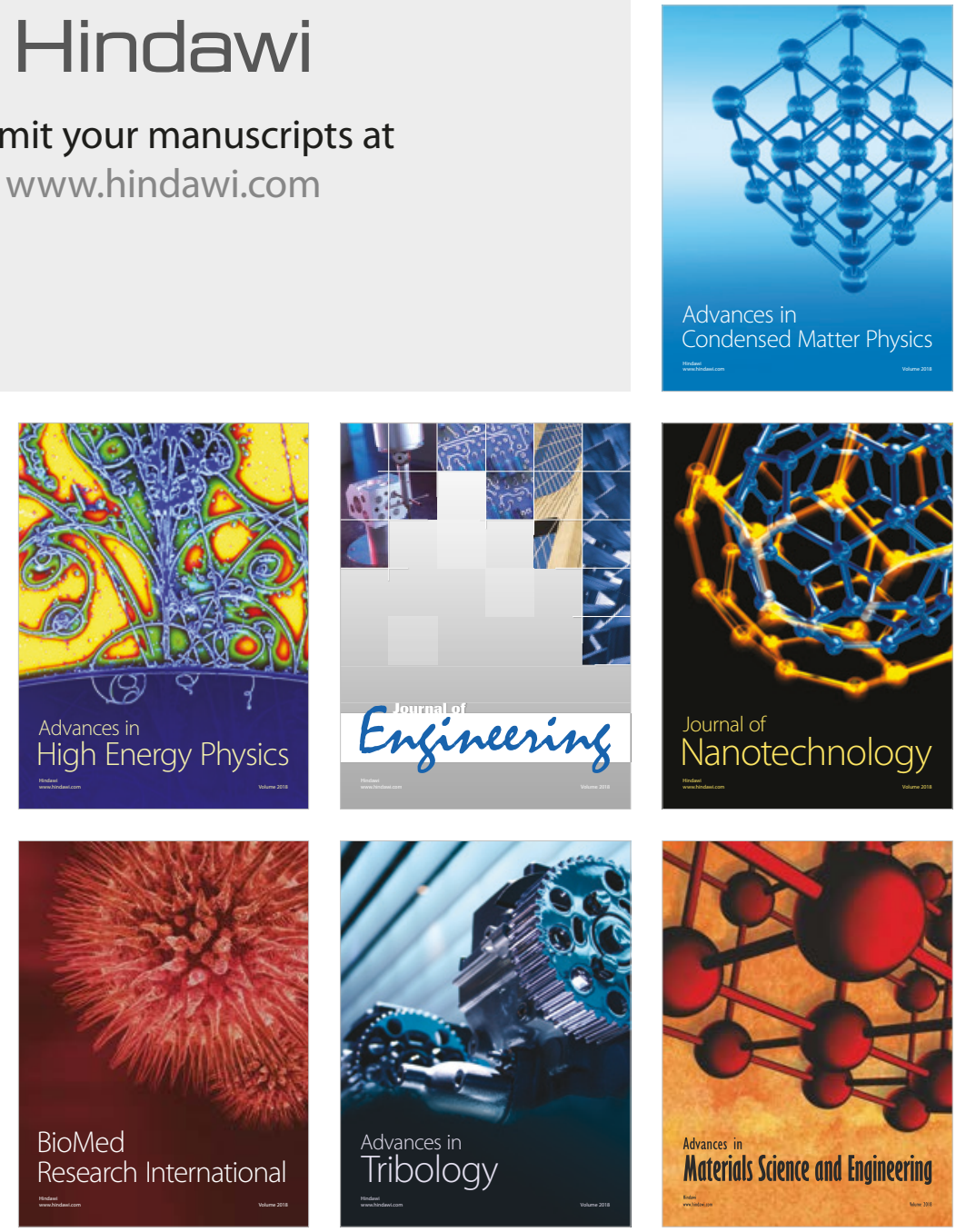